\title{
Numerical and experimental modelling of the centrifugal compressor stage - setting the model of impellers with 2D blades
}

\author{
Richard Matas ${ }^{1, *}$, Tomáš Syka ${ }^{1}$, and Ondřej Luňáček ${ }^{2}$ \\ ${ }^{1}$ University of West Bohemia in Pilsen, New Technologies Research Centre, Univerzitní 8, 30614 Plzeň, Czech Republic \\ ${ }^{2}$ Howden ČKD Compressors s.r.o., Klečákova 347, 19000 Praha 9, Czech Republic
}

\begin{abstract}
The article deals with a description of results from research and development of a radial compressor stage. The experimental compressor and used numerical models are briefly described. In the first part, the comparisons of characteristics obtained experimentally and by numerical simulations for stage with vaneless diffuser are described. In the second part, the results for stage with vanned diffuser are presented. The results are relevant for next studies in research and development process.
\end{abstract}

\section{Introduction}

Centrifugal compressors are widely used in the industry. A traditional producer of multi-stage centrifugal compressors is company Howden ČKD Compressors s.r.o. (HCKD). These compressors are commonly used in many applications across the oil and gas sector, storage and processing, in the metallurgy, mining and also in chemical industry.

The description of processes, operations and performance of the centrifugal compressors are described in many publications, for example in [1]. Performances of the centrifugal compressors stages are analysed namely on account of two main properties: pressure ratio and efficiency. These two parameters vary with volume flow and with Mach number.

A new family of compressor stages is being implemented nowadays. The development project is realized in cooperation with PCA Engineers Limited and University of West Bohemia in Pilsen (UWB). The main goal of the project is to achieve higher stage efficiency and better operational properties. The verification of the new stages properties requires detailed measurement of performance characteristics to obtain reliable data for compressor design. Measured data are also important for validation of CFD numerical models. Validated numerical models are useable for reliable prediction of the stage performance. The validated numerical models can give also the complex information about the flow in the stage and help to design the profile of the channel.

The new stages family consists of stages with $3 \mathrm{D}$ impellers and 2D impellers depending on required flow coefficient. The typical stage with 3D impeller (RTK01) and obtained results are described in [2]. This contribution is focused on stages with 2D impellers. For the study was selected two stages, the first one with vaneless diffuser (RTK04) and the second one with vanned diffuser (RTK06).

\section{Experimental facilities}

\subsection{Experimental rig}

The current development of the centrifugal compressor stages follows the systematic research and development program in the former ČKD company in the past; see for example the paper [3]. The experimental compressor stages verification is being realized in the development test facility HCKD built in Pilsen in 2013, where the single-stage test compressor DARINA IV with the exchangeable flow part is placed. The compressor consists of an inlet cone, an impeller, a diffuser, a return bend, a de-swirl channel and an exit part.

This quite unique compressor is placed on the shared frame with the coupling integrated torquemeter and the gearbox. This machine set is driven by the electromotor with maximum input wattage $1.2 \mathrm{MW}$, which is driven by the frequency converter for the continuous revolution change. The test rig is shown in the upper part of Fig. 1.

The test rig is designed for impellers with diameter of $440 \mathrm{~mm}$. It is possible to change shaft revolutions from 4000 to $17500 \mathrm{rpm}$. A set of throttle-valves for the aerodynamic resistance adjusting is placed in the discharge piping.

\subsection{Experimental equipment}

The parameters of the measured stage are obtained by complex measurement and data acquiring system. Massflow rates of the air through the suction and discharge piping are measured by orifices (max. $42000 \mathrm{~kg}$ /hour).

\footnotetext{
Corresponding author: mata@ntc.zcu.cz
} 


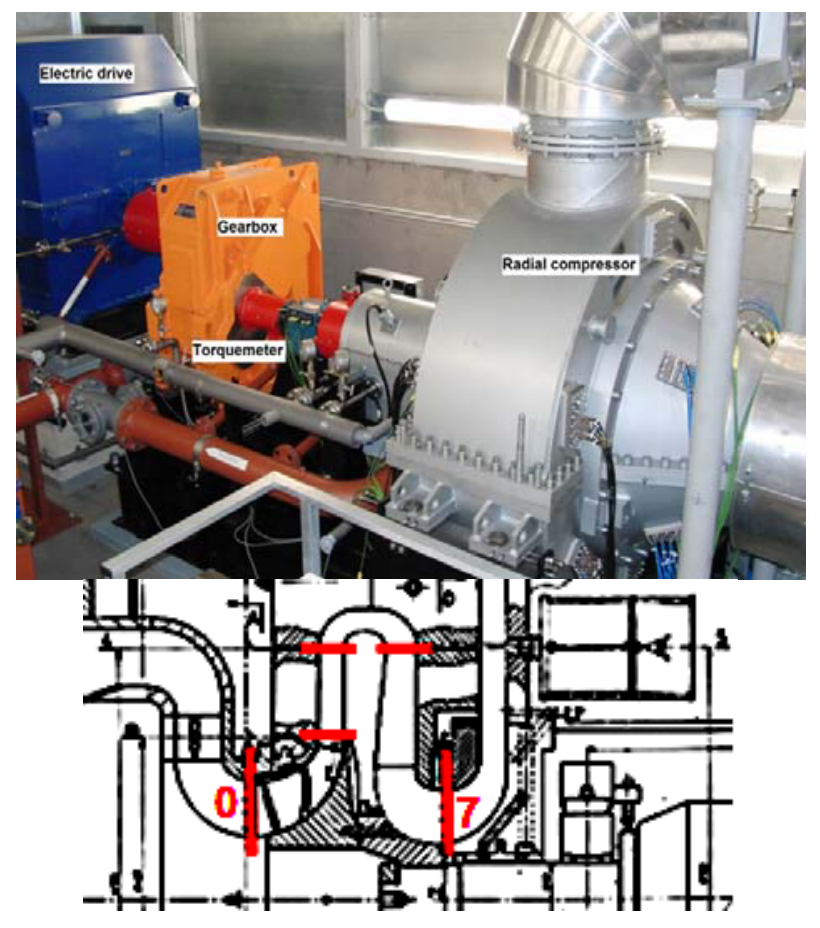

Fig. 1. Centrifugal compressor stage test rig with evaluation planes.

The flow parameters are measured in the evaluation planes as shown in the Fig. 1 down. The description of the planes in the stage is long-term used, see [4]. There is a number of static and total pressure and total temperature probes (multiple sensors are placed in main evaluation planes 0 and 7). Two three-hole pressure probes combined with the positioning system for measuring the return vanes inlet angles are placed in the return channel. The torquemeter gives the value of torque and rotating speed of the compressor rotor.

Physical models of the new designed stages were prescribed in HCKD. The impeller of the stage RTK04 and the vanned diffuser for the stage RTK06 are depicted in the Fig. 2.

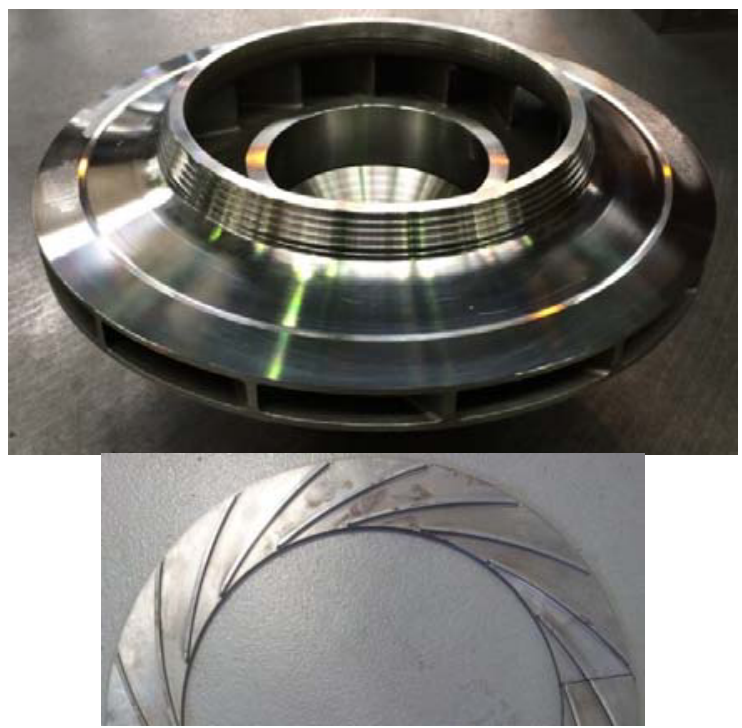

Fig. 2. 2D impeller of the stage RTK04 for experimental compressor and the diffuser vanes for the stage RTK06.

\section{Numerical simulations}

\subsection{Software}

CFD simulations are now an essential tool for the design of all types of devices in turbomachinery. They enable to gain much time between the concept and the test. The verification of simulations is carried out virtually in all development turbomachinery groups and widely published; for example in [5] and many others. The development of the stages has been supported by the CFD simulations in ANSYS/CFX (CFX) and NUMECA FINE/Turbo (NFT) systems.

\subsection{Simulations of stages with 2D impellers}

The methodology of simulation and properties of model has been also evaluated and improved [6]. The mentioned stages were modelled without and with leakage. The geometry of numerical models and computations domains of the stages with leakage can be seen in Fig. 3.
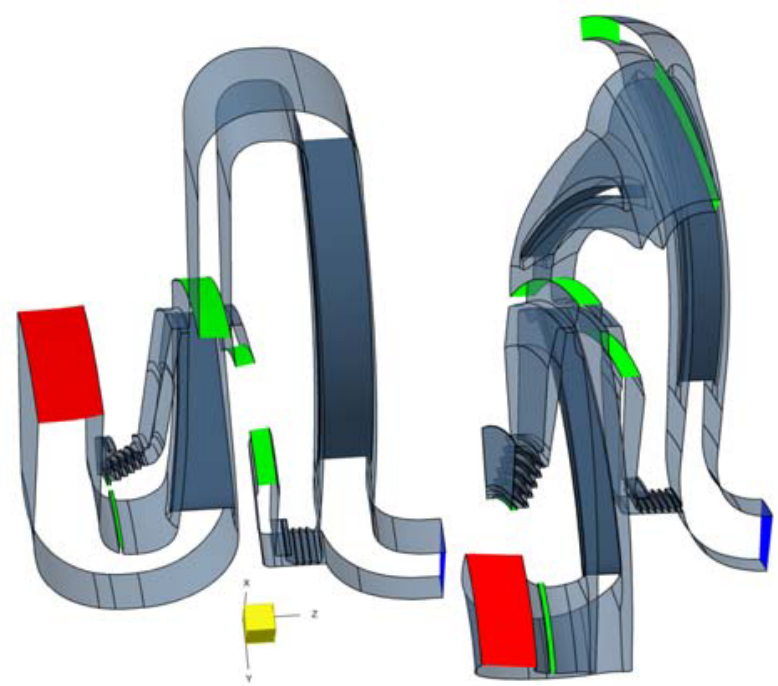

Fig. 3. Stages geometries of the computation domains.

SST k- $\omega$ turbulence model was used for most of the described simulations. For selected simulations of the stage RTK04 was used the EARSM turbulence model in NUMECA FINE/Turbo system.

\subsection{Parameters of stages}

The evaluation of various compressor stages parameters was done according to formulas below. Values of evaluated parameters were averaged by mass flow in postprocessing. The parameters are: specific total work (1), specific isentropic work (2), pressure ratio (3), isentropic efficiency (4) and flow coefficient (5). The indices represent: $t$ - total, $s$ - static, is - isentropic, $p-$ pressure, 0 - plane 0 (stage inlet), 7 - plane 7 (stage outlet), 2 - output from the impeller. 


$$
\begin{aligned}
& \Delta h_{t}=c_{p} \cdot\left(T_{t 7}-T_{t 0}\right) \\
& \Delta h_{i s}=z \cdot r \cdot T_{t 0} \cdot \frac{\kappa}{\kappa-1}\left[\left(\frac{p_{t 7}}{p_{t 0}}\right)^{\frac{\kappa-1}{\kappa}}-1\right] \\
& P I=\frac{p_{t 7}}{p_{t 0}} \\
& E T A=\frac{\Delta h_{i s}}{\Delta h_{t}} \\
& F I 0=\frac{4 \cdot Q_{0}}{\pi \cdot D_{2}^{2} \cdot u_{2}}
\end{aligned}
$$

The obtained characteristics include the confidental data of HCKD and only relative values are presented in the next chapters consequently. The values $P I_{r}, E T A_{r}$ and $m_{r}$ are related to the design point in the measured characteristic.

\section{Comparison of results - stage RTK04}

The results from CFD stages simulations were compared to the measured data converted to the standard inlet conditions. The Fig. 4 shows the computational mesh for NFT simulations with leakage. The numbers of cells for CFD models are shown in the Table 1.

Table 1. Numbers of cells for CFD models of RTK04.

\begin{tabular}{|c|c|}
\hline CFD model & Number of cells \\
\hline CFX & $1,653,523$ \\
\hline CFX with leakage & $2,695,255$ \\
\hline NFT & $2,996,000$ \\
\hline NFT with leakage & $6,056,000$ \\
\hline
\end{tabular}
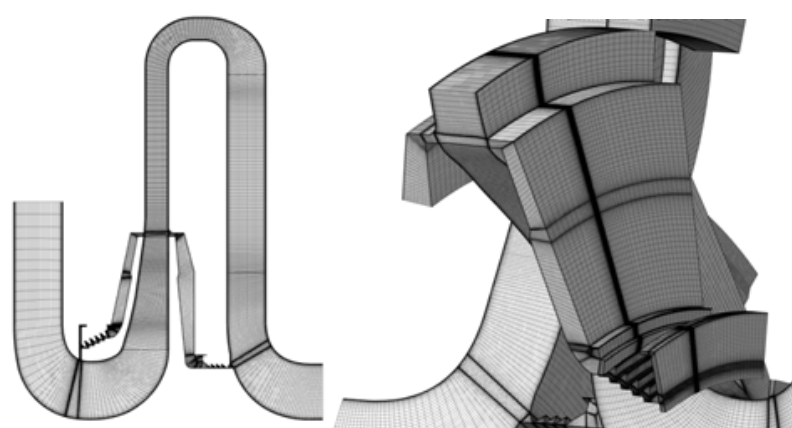

Fig. 4. Computational mesh of the RTK 04 stage (NFT)

The Fig. 5 shows the averaged pressure distribution in the meridional view. In the Fig. 6 you can see the performance characteristics. The Fig. 7 depicts the inlet flow angle into return channel vanes. Both figures are for $12666 \mathrm{rpm}$. It is possible to compare the results of described CFD simulations with measured data.

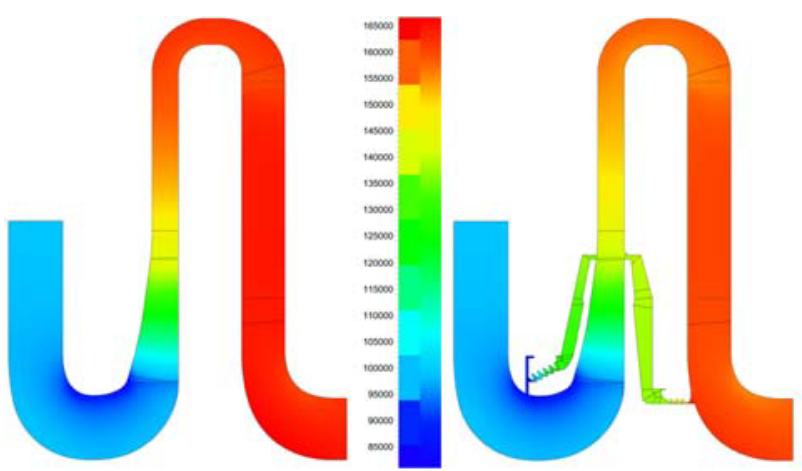

Fig. 5. Pressure distribution $[\mathrm{Pa}]$ in the stage RTK04 with and without leakage for the design point - meridional averaging

The results of both CFD systems are similar. The results of the NFT cases with the EARSM turbulent model are very close to the measured values of $P I$ and ETA. This configuration of the CFD model can be used for reliable simulations of radial compressor stage characteristics of similar geometric and flow parameters. The evaluated values in models without leakage are overpredicted.
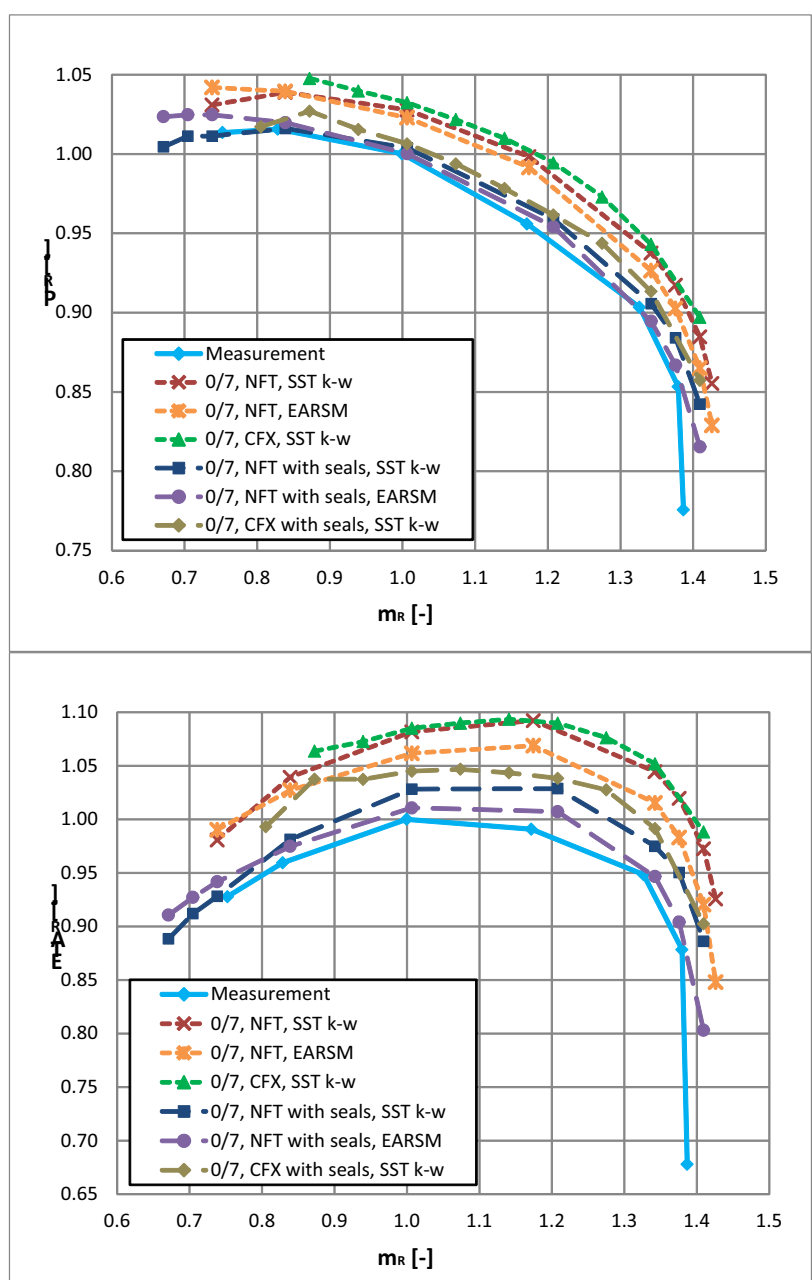

Fig. 6. Performance characteristics of the RTK 04 stage

The inlet flow angles into return channel vanes are very similar for all computed models. The values for higher mass flows are satisfying for simulations in comparison to measured values. The comparison for 
lower values of mass flow shows that the values in experiments rise, whereas in simulations sink. The reason can be the unsteady phenomena in the flow field or asymmetric flow in various channels which can't be detect in one channel model of the stage.

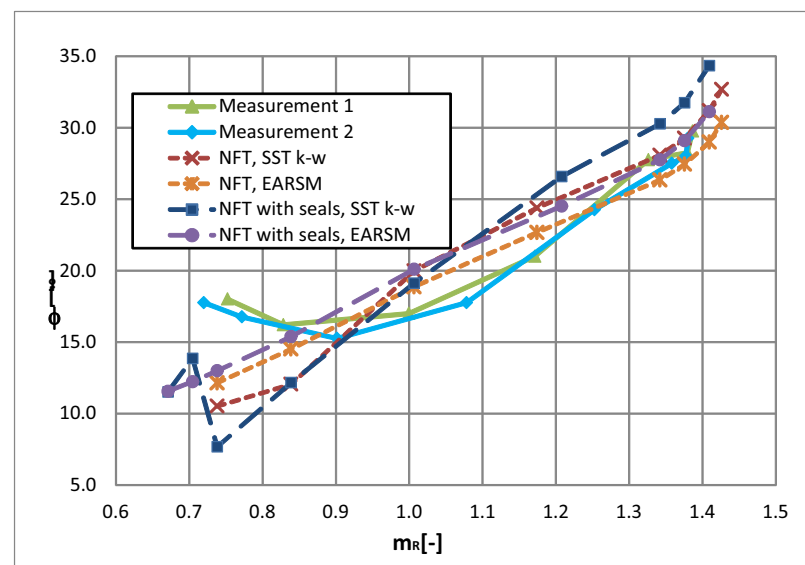

Fig. 7. Evaluation of the inlet flow angles into return channel vanes of the RTK 04 stage

\section{Comparison of results - stage RTK06}

The same process as for the stage RTK04 was implemented for the stage RTK06. The numerical model is more sophisticated due to vanned diffuser, because the model of the diffuser vane has to be added.

The Fig. 8 shows the computational mesh for NFT computations with leakage. The numbers of cells for CFD models are shown in the Table 2.

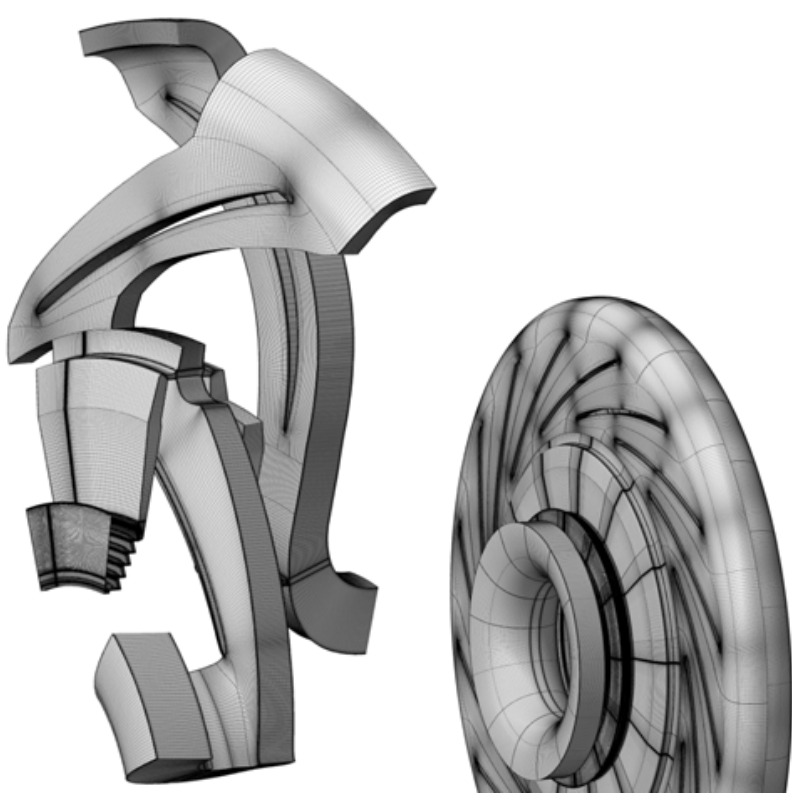

Fig. 8. Computational mesh of the RTK 06 stage (NFT and CFX II with leakage)
Table 2. Number of cells for numerical models of RTK06.

\begin{tabular}{|c|c|}
\hline CFD model & Number of cells \\
\hline CFX I & 885,660 \\
\hline CFX I with leakage & $1,132,269$ \\
\hline NFT and CFX II & $6,120,000$ \\
\hline $\begin{array}{c}\text { NFT and CFX II with } \\
\text { leakage }\end{array}$ & $11,070,000$ \\
\hline
\end{tabular}

The Fig. 9 shows the flow field in the meridional view. In the Fig. 10 are presented the performance characteristics. The results of numerical simulations can be compared sufficiently to the measured values.

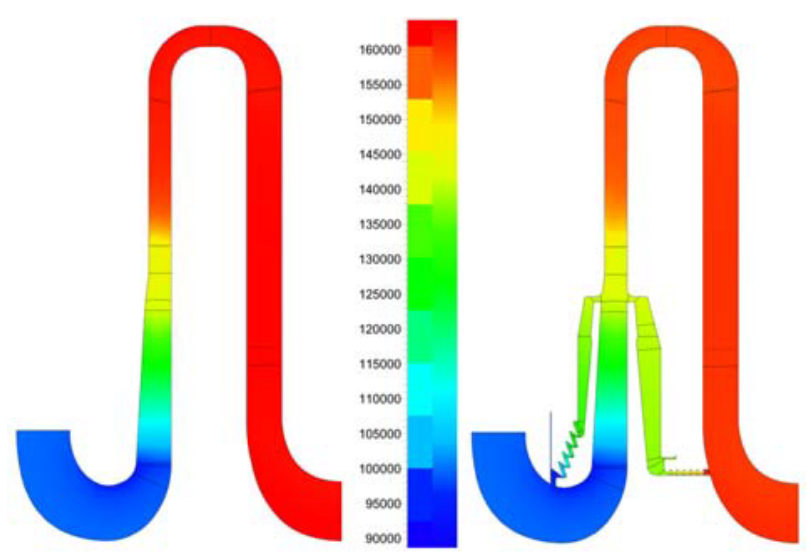

Fig. 9. Pressure distribution $[\mathrm{Pa}]$ in the stage RTK06 with and without leakage for the design point - meridional averaging

The performance characteristics in models without leakage are overpredicted again. The results evaluated from coarse mesh simulations show relatively high deviation from measured values. The results of both CFD systems with leakage and fine meshes are similar. The results of these cases are very close to the measured values of $P I$ and ETA. These configurations of the CFD models can be used for the reliable simulations of characteristics for radial compressor stages with vanned diffusers of similar geometric and flow parameters. The disadvantage is relatively complicated mesh generation and relatively high computational costs.

\section{Conclusions}

The verification of the compressor stages with the relatively wide range of parameters is possible to perform on the HCKD DARINA IV compressor with good repeatability. The test facility is able to evaluate complete integral and some local quantities in order to prepare appropriate data for thermodynamic design. It is possible to verify CFD models and their applicability by mutual comparison of experimental data and CFD simulations results. 

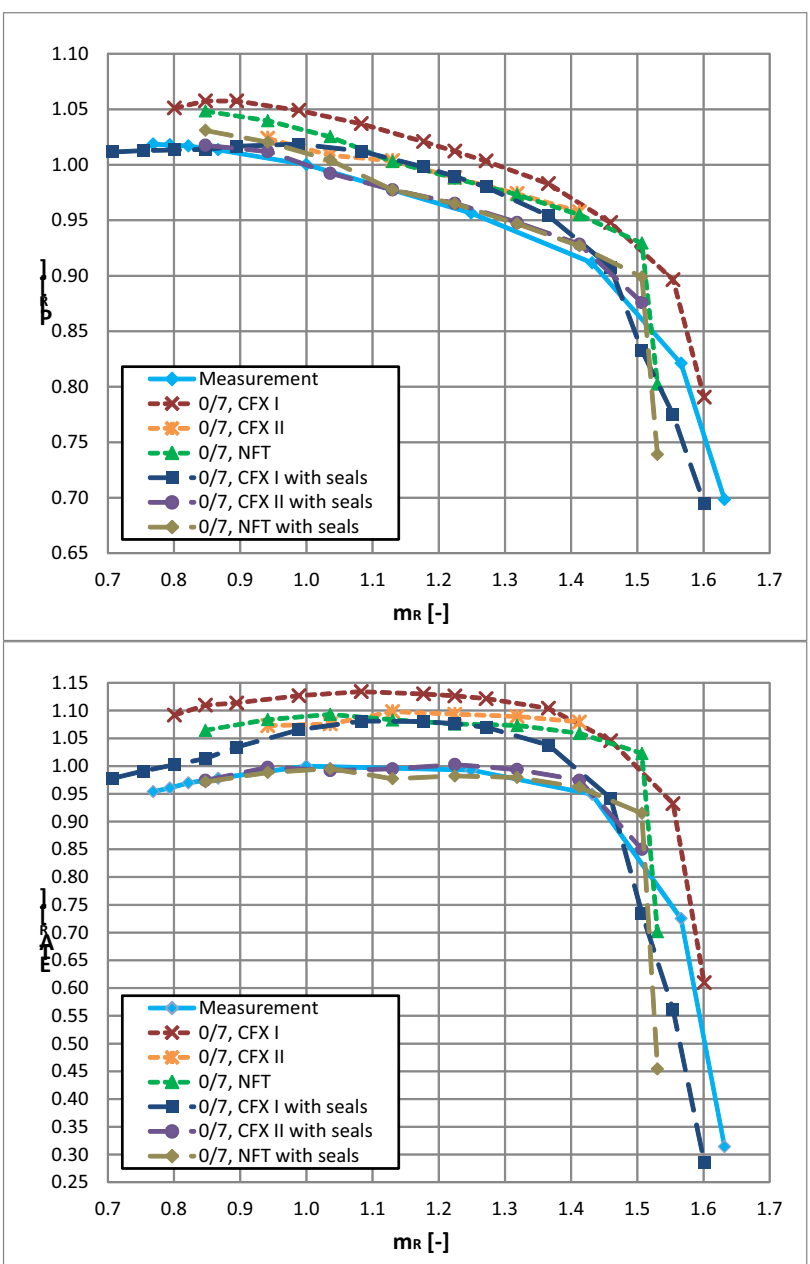

Fig. 10. Performance characteristics of the RTK 06 stage

The methodology for the centrifugal compressor stages CFD simulations was verified in the NUMECA FINE/Turbo and ANSYS/CFX CFD software. Based on the verified numerical simulations, it is possible to additionally enhance experimental measurement and also improve the quality of the development process, because CFD results cover complete quantities distribution in the stage. Beside this, it also enables to simulate various modifications of stage quickly and to assess them without the need of excessive testing.

The values from models without leakage are overpredicted. For 2D impellers are necessary to use correct coefficients for using the results in the development process. The benefit of these models is relatively rapid generating of the computational meshes and low computational costs.

The presented analyses of the $2 \mathrm{D}$ stages and the verifications of the numerical models are the first steps for further works. In the case of the stage RTK04 it is the study, which has been focused on the influence of the technological holes for welding in various positions on the rotor blades to the stage characteristics. The stage RTK06 served to the experimental and numerical modelling of the leakage clearances and influences of their values on the stage characteristics. Results of both mentioned studies will be published subsequently.
The results were developed within the CENTEM PLUS (LO1402) by financial means from the Ministry of Education, Youth and Sports under the "National Sustainability Programme I." and in the framework of the FR-TI3/421 project (Ministry of Industry and Trade of the Czech Republic) and specific research. The authors would like to thank the Howden CKDD Compressors Ltd. for permission to publish this paper and all colleagues who participated on this work.

\section{References}

1. M. P. Boyce, "Principles of Operation and Performance Estimation of Centrifugal Compressors," Proceedings of the twenty-second turbomachinery symposium, Dallas, TX. (1993)

2. Numerical and Experimental Modelling of the Radial Compressor Stage Syka, Tomáš and Matas, Richard and Luňáček, Ondřej, AIP Conference Proceedings, 1745, 020059 (2016).

3. J. Paroubek and J. Kynčl, "The Influence of Impeller Flow Channel on Aerodynamic Performance of a Centrifugal Compressor Stage," ASME Paper 98-GT-40 (1998).

4. J. Paroubek and V. Cyrus and J. Kynčl, „The Influence of Outlet Stator Part Surface Roughness on the Performance of a Very Low Flow Coefficient Centrifugal Compressor Stage", ASME Paper 96-OT-179 (1996)

5. P. Le Sausse and P. Fabrie and D. Arnou and F. Clunet, "CFD comparison with centrifugal compressor measurements on a wide operating range," EPJ Web of Conferences, Volume $\mathbf{4 5}$ (2013)

6. T. Syka, "Geometry structure influence on the radial compressor stage efficiency," $\mathrm{Ph} . \mathrm{D}$. thesis, in Czech, ZČU in Pilsen (2015) 